\title{
LA EDUCACIÓN Y LOS EMPRESARIOS EN ANDALUCÍA
}

Felipe Rafael CÁCERES CARRASCO*

\section{INTRODUCCIÓN}

Desde hace ya algunos años se percibe en los países europeos una creciente preocupación por el fenómeno de la emergencia empresarial. Expresiones similares a "cultura empresarial o emprendedora", "emprendedores", "iniciativa empresarial", etc. son a menudo pronunciadas por políticos o ciertos sectores de la población para hacer referencia, en numerosas ocasiones con insuficiente precisión, a valores, comportamientos, actitudes o rasgos que se identifican con la empresarialidad.

Los cambios que se han producido en las economías europeas y en las políticas económicas que en ellas se han ejecutado desde mediados de los años setenta hasta nuestros días, como el estancamiento o incluso retroceso en el crecimiento relativo del sector industrial, el problema del desempleo, la importancia de las PYMES en la generación de puestos de trabajo, el avance de las políticas liberales frente a als intervencionistas, etc. han tenido, entre otras consecuencias, un resurgir de la figura del empresario individual como agente económico capaz de crear riqueza y empleo. No es extraño, por tanto, que políticos e investigadores hayan intentado avanzar en el conocimiento de las causas que explican la aparición de empresarios y su calidad empresarial.

En el caso de Andalucía dichos análisis adquieren un interés especial si se tiene en cuenta que con cierta frecuencia, diversos autores, han señalado entre los factores que explican el retraso económico de la región frente a otras áreas geográficas españolas la existencia de un supuesto bajo nivel de espíritu empresarial. Encontrar las razones que explican la oferta de empresarios en una economía puede ser una tarea de gran dificultad. Sin embargo, algunos autores han venido señalando algunos factores que pueden favorecer la emergencia empresarial. Uno de éstos es el nivel de instrucción.

* Departamento de Economía Aplicada I. Universidad de Sevilla. 


\section{EDUCACIÓN Y EMPRESARIOS}

Una cuestión impícita o explícita que necesariamente aparecerá cuando se trata de analizar el papel que juega la educación en la oferta de empresarios es si dichc agente nace o se hace. Diferentes autores han ofrecido respuestas controvertidas que, sin embargo, si se analizan con detenimiento permiten una cierta aproximación en los resultados que de ellas se derivan.

Por un lado pueden situarse los autores que, como González Simón, consideran que el talento es una cualidad innata fundamental para el éxito empresarial. ya que sin él no es posible ni la sagacidad ni la intuición, características personales sin las que no concibe al empresario. Por tanto, desde esta perspectiva, una especial educación, aunque pueda resultar útil, no es fundamental para el empresariado. Y a ese respecto, González señala que muchos de los grandes empresarios abandonaron la escuela a una edad muy temprana mientras que otros interrumpieron sus estudios universitarios antes de finalizarlos ${ }^{1}$.

Desde otra óptica, Schultz, que conceptúa como empresario a toda persona que es capaz de tratar con las situaciones de desequilibrio, señala que la oferta de capacidad empresarial se incrementa inconfundiblemente con la escolarización adicional, al ejercer ésta un claro efecto favorable sobre la capacidad para hacer frente a los desequilibrios asociados con la modernización económica. Por lo que propone la inversión en educación como medio para aumentar la oferta de empresarios y lograr así un mayor desarrollo económico ${ }^{2}$.

Las dos posturas anteriores parecen representar posiciones muy diferentes, sin embargo, los resultados a que conducen pueden aproximarse considerablemente. Ciertamente, en la primera de ellas se hace referencia a cualidades innatas, independientes, por tanto, de la formación. No obstante, su autor señala asimismo que las tendencias del individuo pueden ser reforzadas y elaboradas, o confundidas, contradichas y embotadas por los modelos de comportamiento que fijen los profesores, contribuyendo la educación a que el individuo tenga mayor confianza en si mismo o sea menos ansioso al enfrentar nuevos problemas. De igual modo, González Simón señala que un incremento de los tipos de educación apropiados eleva la capacidad de los individuos para concebir y establecer nuevas empresas productivas, ampliando así la gama de las que éstos están preparados para concebir y establecer ${ }^{3}$. Por tanto, es posible deducir que con independencia de que el talento empresarial sea un rasgo innato o adquirido, la educación ejercerá un efecto positivo sobre la oferta empresarial, ya que incluso en el caso de que se aceptara la postura, poco acertada bajo nuestro punto de vista, de que el talento empresa-

1. González Simón, M. (1992): "Educación y Empresarios". VI Reunión ASEPELT-ESPAÑA, Granada.

2. Schultz, T: (1985): Invirtiendo en la gente. Editorial Ariel, Barcelona, págs. 37-38.

3. González Simón, M. (1992): op. cit. 
rial es algo innato, la educación, al elevar la capacidad de concebir y establecer nuevas empresas productivas aumentará las posibilidades de creación de éstas reforzando y favoreciendo así las tendencias de los individuos con buenas cualidades empresariales hacia la actividad empresarial.

Abundando en lo anterior puede señalarse que algunos investigadores han encontrado una correlación positiva entre nivel de formación y orientación hacia la actividad empresarial. Es decir, que la preferencia por el empresariado como carrera profesional se incrementa a medida que se eleva el nivel de formación, aunque es preciso señalar que dicha preferencia viene marcada, en gran medida, por el tipo de educación recibida. Siendo notable a ese respecto la influencia de la Licenciatura en Ciencias Económicas y Empresariales.

Por tanto, todo lo señalado en los párrafos anteriores apunta a que el nivel de educación ejerce un efecto positivo sobre la orientación de los individuos hacia la actividad empresarial, que se ve incrementado cuando éstos cursan ramas que se identifican directamente con la empresa. Ahora bien, algunas investigaciones ban puesto de manifiesto que los efectos de la formación sobre la emergencia y calidad empresarial pueden incrementarse considerablemente mediante la aplicación, por parte de los profesores, de una metodología orientada a ese objetivo.

A ese respecto se ha señalado que la educación para empresarios no debe centrarse solamente en los conocimientos teóricos; que un elemento crucial de ésta debe ser la práctica, pues difícilmente podrán transmitirse a través de la teoría determinados conocimientos tácitos que son relativamente fácil de adquirir cuando la enseñanza se realiza bajo condiciones que reflejen, lo mejor posible, el ambiente de incertidumbre en el que se desenvuelve el empresario.

Así, Gibb, considera que el modelo para aprender empresarialidad debe contemplar tres tipos de influencias:

1. Los refuerzos esenciales del empresario en la organización.

2. Una estructura que soporte las decisiones tomadas bajo incertidumbre.

3. Un modo de aprendizaje centrado en el autodescubrimiento.

De igual modo señala Gibb, que dicho modelo debe reunir:

1. Las esencias de la persona emprendedora dentro del ambiente de Ia clase, lo que viene a suponer que los estudiantes gozan del mayor control posible en un ruarco de libertad y flexibilidad en el que se puede aprender de los errores y se permite la informalidad y el experimento para dar solución a los problemas que se presenten.

4. Guzmán, J. (1995): El Empresariado en la Provincia de Sevilla. Diputación Provincial de Sevilla, Sevilla. págs. $165-176$. 
2. Un proyecto de tareas de "manager" que se utilizará para aprender bajo condiciones de incertidumbre. Se trata de simular actividades operando en un ambiente incierto. Con este método, el estudiante debe dirigir todas las tareas del proyecto a realizar, lo que le obligará a desarrollar ideas, evaluarlas, planificar, considerar de los recursos, llevar a cabo el proyecto y realizar un "feed back".

3. Un modo de enseñar, que ha de suponer un estilo opuesto a una didáctica formal. Esencialmente el papel del profesor es el de guía y compañero en el proceso de aprendizaje, y se centra sustancialmente en las distintas formas de cómo aprender, entendiendo dicho proceso y facilitándolo.

Para Gibb, la combinación de estos tres elementos estimulará los comportamientos emprendedores y las destrezas y atributos asociados a ellos ${ }^{5}$. Es decir, este tipo de formación, con independencia de que trate de transmitir ciertos conocimientos formalizables, va dirigido a desarrollar o estimular un conjunto de características personales del individuo que se asocian con la empresarialidad, lo que favorecerá no sólo la aparición de nuevos empresarios sino también su calidad.

Por otro lado, Johannisson, para quien la innovación es un factor fundamental en el empresariado, considera que la formación en una clase puede arruinar la única capacidad del empresariado para la creación y desarrollo de nuevas empresas, por lo que propone un cambio de la metodología tradicional en las universidades y colegios; instituciones, que, a su juicio, deberían adoptar las metodologías que se aplican en las escuelas de arte, pues, según señala, el empresariado es más un arte que una ciencia. Desde esa perspectiva, pone de relieve la importancia que tienen, para el aprendizaje del empresariado, los intercambios entre los centros de enseñanza y las empresas, destacando cómo las prácticas en empresas son un elemento crucial para la adquisición de competencias empresariales distintas de los conocimientos teóricos ${ }^{6}$.

En conclusión, a partir de lo expuesto en este epígrafe puede señalarse que el nivel de educación es una variable que puede influir positivamente sobre la orientación de los individuos hacia las actividades empresariales. Asimismo, se ha puesto de manifiesto que existe un tipo de formación, que, a diferencia de la que se podría denominar "standard", va dirigida, mediante una metodología específica, a estimular o desarrollar características psicológicas de los individuos que se asimilan a la empresarialidad, por lo que sus efectos sobre la calidad y emergencia empresarial son mayores.

5. Gibb, A. (1993): "The Enterprise Culture and Education. Understanding Enterprise Education ands its Links with Small Business. Entrepreneurship and Wider Educational Goals". International Small Business Joumal, núm. 11 , vol. 3 .

6. Johannissson, B. (1991): “University Training for Entrepreneurship: Swedis Approaches", Entrepreneurship and Regional Development, núm. 3 


\section{FORMACIÓN Y EMPRESARIOS EN ANDALUCÍA}

Según se desprende del análisis realizado en el epígrafe anterior el nivel de formación puede incidir sobre la orientación de los individuos hacia la actividad empresarial. Sin embargo, cuando se observan los datos relativos al nivel de formación de los empresarios andaluces puede apreciarse que esas influencias han sido bastante limitadas.

Los datos del cuadro I proceden de una encuesta realizada entre 1997 y 1998 a empresarios de Andalucía Occidental sobre una muestra de 278 individuos, distribuida proporcionalmente según sector de actividad, excepto el agrícola, y dimensión empresarial (número de empleados). Como puede apreciarse, casi la mitad de los empresarios andaluces inició su primera empresa con un nivel de formación primario o muy elemental y tan sólo un $9 \%$ lo hizo siendo licenciado, lo que, en principio, no parece corresponderse con los resultados del análisis desarrollado en el epígrafe anterior. No obstante, cabría señalar que una de las razones que explican los valores que recoge la tabla es la dificultad que una gran mayoría de esos empresarios, que iniciaron su primera empresa en los últimos cuarenta años aproximadamente, tuvieron para adquirir una formación media o superior dado que el acceso a los institutos o universidades no ha sido posible en Andalucía para muchas familias hasta hace no demasiados años.

\section{CUADRO I}

\section{FORMACIÓN DE LOS EMPRESARIOS ANDALUCES EN LOS INICIOS}

\begin{tabular}{lcr}
\hline Nivel de Formación & № de Empresarios & $\%$ \\
\hline Primaria o Ninguna & 122 & 43,9 \\
Bachillerato & 57 & 20,5 \\
F.P. Primer Grado & 10 & 3,6 \\
F.P. Segundo Grado & 22 & 7,9 \\
Diplomado & 42 & 15,1 \\
Licenciado & 25 & 9,0 \\
\hline Totales & 278 & 100,0
\end{tabular}

Fuente: Elaboración propia.

Una manera de valorar la importancia que sobre la formación del empresariado ha tenido dicha circunstancia histórica puede consistir en comparar como varían los niveles de formación de los empresarios cuando se distingue entre los que crearon su primera empresa en los últimos cinco años y los que se hicieron empresarios con anterioridad a ese periodo. 
Los datos que aparecen el cuadro II permiten afirmar que la formación de lo: empresarios emergidos en los últimos cinco años se ha elevado en los tramos me dios aunque resulta llamativo observar que ha disminuido cuando se trata de la for mación superior (diplomatura y licenciatura). Asimismo, pese a dicha mejora er los niveles medios, todavía en los últimos años un elevado número de empresa. rios han emergido con formación primaria. De igual forma, se aprecia que el gru. po de empresarios que creó su primera empresa con nivel educativo de Formaciór Profesional es relativamente bajo, sobre todo si se compara con el de aquellos que iniciaron su actividad con formación básica, lo que parece ciertamente contradic. torio si se tiene en cuenta la estrecha relación que muchas de las ramas de ese tipc formación tienen con las actividades empresariales.

\section{CUADRO II \\ FORMACIÓN AL INICIAR SUS NEGOCIOS SEGÚN AÑO DE SU PRIMERA EMPRESA}

\begin{tabular}{lcccccc} 
N" Años & $\begin{array}{c}\text { Primaria } \\
\text { o Ninguna }\end{array}$ & Bachill. & F.P. 1\% G. & F.P. 2" G. & Diplom. & Licenc. \\
\hline$<6(\mathrm{n}=68)$ & $20(29,4 \%)$ & $19(27,9 \%)$ & $6(8,8 \%)$ & $8(11,8 \%)$ & $8(11,8 \%)$ & $7(10,3 \%)$ \\
$>5(\mathrm{n}=210)$ & $202(48,6 \%)$ & $38(18,1 \%)$ & $4(1,9 \%)$ & $14(6,7 \%)$ & $34(16,2 \%)$ & $18(8,6 \%)$ \\
\cline { 2 - 7 } Totales & $122(43,9 \%)$ & $57(20,5 \%)$ & $10(3,6 \%)$ & $22(7,9 \%)$ & $42(15,1 \%)$ & $25(9,0 \%)$
\end{tabular}

Fuente: Elaboración propia.

Por otro lado, el análisis anterior puede completarse observando el peso de los empresarios que antes de iniciar su primer negocio habían realizado algún curso que al margen de los conocimientos formales que en él se impartieran, estuviera dirigido a estimular las actitudes y comportamientos empresariales mediante metodologías apropiadas para ello, tal como se expuso en el epígrafe anterior. Asimismo, puede resultar de interés valorar la importancia relativa del grupo de empresarios emergidos en Andalucía que habían recibido algún tipo de formación relacionada con la actividad de la empresa que crearon con anterioridad a su «debut» como tales (cuadro III).

Como puede observarse, la formación específica para empresarios, aquélla que incide sobre actitudes y comportamientos asociados a la empresarialidad, ha sido prácticamente nula entre los empresarios que han emergido en Andalucía Occidental. Asimismo, se aprecia que tan sólo un $10 \%$ de dichos agentes habían recibido cursos de formación relacionados con la actividad de la empresa que crearon antes de poner en marcha a ésta. 
CUADRO III

EMPRESARIOS CON FORMACIÓN EMPRESARIAL PREVIA

Tipo de Formación

Formac. Empresarial

Formac. Actividad

Ninguna

Totales
$\mathrm{N}^{2}$ de Empresarios

7

30

241
$\%$

10,8

86,1

Fuente: Elaboración propia.

Se constata así que la formación ha ejercido una influencia limitada en la emergencia de los empresarios de Andalucía Occidental, siendo especialmente llamativo que el número de empresarios emergidos en los últimos cinco años con niveles educativos de Formación Profesional o de enseñanza superior no haya crecido de forma más rápida respecto a los empresarios que crearon su empresa hace más de cinco años, pues, como es sabido, en las últimas décadas se han incrementado notablemente para la población andaluza las posibilidades de acceso a esos niveles formativos.

El análisis anterior pone de manifiesto la posibilidad de que el sistema educativo no esté respondiendo del modo adecuado a una de las necesidades que, desde diferentes instancias políticas y económicas, se ha señalado en relación con la economía andaluza: la mejora del tejido empresarial de la Comunidad Autónoma. Una necesidad que tiene su expresión, entre otros, en los siguientes hechos: reducida dimensión de las empresas, bajo nivel de articulación productiva en el conjunto de la región, reducida tasa de innovación empresarial, excesiva orientación comercial hacia los mercados más próximos, ausencia o baja presencia de actividades de alto nivel tecnológico, excesiva concentración de la industria en torno pocos centros. Por supuesto, que superar todos esos handicaps no puede ser labor exclusiva de la formación, pero el limitado papel que ésta parece estar jugando en la emergencia de un empresariado de calidad puede llegar a representar un importante obstáculo para avanzar hacia la superación de esos problemas. Es decir, que se están desaprovechando unos recursos que, en la actual situación de desempleo en Andalucía, podrían incrementar la eficacia de las políticas que persiguen el crecimiento económico de la comunidad autónoma y la generación de puestos de trabajo, dado que, según se ha expuesto en el epígrafe anterior, la formación, sobre todo en las titulaciones que más se relacionan con la empresa, podría orientarse en mayor medida a estimular la aparición de empresarios así como a elevar la calidad de éstos. 
Ahora bien, si el papel de la educación en la emergencia empresarial andaluz: ha sido tan escaso como parece revelar la información anterior cabe preguntars donde han adquirido los empresarios emergidos en Andalucía, muchos de ellos cor unos bajísimos niveles de formación antes de crear su primera empresa como se hi visto, los conocimientos necesarios para la puesta en marcha y desarrollo de st negocio. La respuesta quizás pueda encontrarse en el cuadro IV, donde se recoger. los años de experiencia laboral previa a la puesta en marcha del negocio propio que los empresarios emergidos en Andalucía Occidental habían acumulado.

\section{CUADRO IV}

AÑOS TOTALES DE EXPERIENCIA LABORAL

\begin{tabular}{lcr}
\hline № Años de Experiencia & № de Empresarios & $\%$ \\
\hline 0 Años & 9 & 3,2 \\
Hasta 2 Años & 36 & 12,9 \\
Entre 3 y 5 Años & 50 & 18,0 \\
Entre 6 y 10 Años & 73 & 26,3 \\
Más de 10 Años & 110 & 39,6 \\
Totales & 278 & 100,0 \\
\hline
\end{tabular}

Fuente: Elaboración propia.

Los datos revelan que tan sólo un 3,2\% de los empresarios andaluces no habían desarrollado experiencia laboral alguna antes de crear su primera empresa, mientras que un $66 \%$ había desarrollado actividades laborales en alguna organización durante un periodo de tiempo superior a cinco años. Puede resultar de interés completar esa información señalando que la experiencia laboral media de los individuos muestreados, con anterioridad a su aparición como empresarios, ha sido de 10,5 años, lo que unido a los resultados anteriores permite afirmar que en el caso de Andalucía ha sido la experiencia laboral, más que la formación, el factor que ha incidido en la aparición de los empresarios. O si se quiere de otra manera, puede decirse que en Andalucía la formación que más ha estimulado la emergencia empresarial es la que se ha adquirido por medio de la experiencia laboral. La empresa parece haberse convertido en la principal «escuela para empresarios» supliendo así, con todas sus ventajas e inconvenientes, las deficiencias del sistema educativo en lo que se refiere a orientar a los individuos hacia la creación de nuevas empresas.

De todo ello se deduce que, al contrario de lo que algunos enfoques teóricos mantienen, la educación no ha ejercido un marcado efecto positivo en la aparición 
del empresario andaluz, lo que permite colegir que el sistema educativo andaluz ofrece grandes potencialidades para contribuir a mejorar el tejido empresarial de la región que no están siendo explotadas adecuadamente.

\section{EL PAPEL DE LA FORMACIÓN EN LA EMERGENCIA EMPRESARIAL ANDALUZA}

A partir de lo expuesto en el punto anterior cabe preguntarse cuál debe ser el papel que la Universidad y los Centros de Enseñanza Media deben jugar en el desarrollo de la función empresarial para contribuir, desde esa perspectiva, a lograr un mayor crecimiento económico. La siguiente cita de Sarabia, aunque escrita hace ya varios años, permite, por su actualidad para la universidad andaluza, señalar algunos aspectos de interés a este respecto:

"la universidad está empezando a contribuir al desarrollo empresarial con más y mejor formados profesionales, pero sinceramente creo que la universidad no está hoy en condiciones de transmitir esa parte del espíritu de empresario capaz de asumir el riesgo propio, es decir, esa inquietud por crear nuevos centros de actividad económica, esa capacidad para jugarse dentro de un mercado libre y abierto lo que hasta el momento se ha conseguido, por lo general con esfuerzo y sacrificio. El estudiante no percibe de la mayoría de sus profesores esa afición por el riesgo, ni tampoco la creatividad es una de las facultades que más se potencian en nuestra universidad; por lo general nuestros estudiantes sólo adquieren conocimientos y técnicas"7.

Desde esa perspectiva Sarabia parece señalar que la universidad sólo forma en una de las dos dimensiones que están relacionadas con las funciones que desarrolla el hombre empresario; razón por la que García Echevarría reclama la necesidad de que la universidad forme en dos dimensiones diferentes que se relacionan con:

- La función empresarial propiamente dicha, y

- La directiva.

En relación con la primera, García Echevarría, destaca que el empresario ha de tener capacidad y conocimientos para integrar las funciones y a los expertos, y de esta manera dar respuesta a situaciones cambiantes del entorno. En cuanto a la función directiva, implica otro tipo de conocimiento y otro tipo de acción y comportamiento. Por tanto, a su juicio, la universidad debe formar:

7. Sarabia, A. (1988): "La Universidad y su influencia en el Desarrollo Empresarial". El empresario en la Sociedad Actual, II Congreso Mundial Vasco, pág. 70. 
- En la dimensión de una capacidad integradora, globalizante, es decir, «ls dimensión corporativa y el hombre responsable de la misma».

- En la dimensión directiva funcional, que supone la transmisión de un con. junto profundo de conocimientos que harán posible a quien los posea asu. mir la tarea de asignación de recursos y de contribuir al éxito o fracaso $\mathrm{d} \epsilon$ la empresa ${ }^{8}$.

Quizás un modo más concreto de distinguir entre esas dos dimensiones de le formación pueda ser diferenciando entre:

- Conocimientos formalizables.

- Conocimientos no formalizables.

Los primeros son los que habitualmente se transmiten por la formación standard, se trata de técnicas, métodos y conocimientos que al admitir su formalización pueden ser transmitidos con relativa facilidad. En cambio, los no formalizables se caracterizan porque están estrechamente conectados con praxis, las actitudes y las características personales de los empresarios. Son apropiados para desarrollar funciones que se enmarcan en un contexto de incertidumbre, como pueden ser la asunción de riesgos, la movilización de hombres y medios en la dirección deseada, la captación de oportunidades de negocio, la innovación o las grandes decisiones estratégicas, en las que la capacidad de adelantarse a un futuro inestimable por medios estadísticos con un mínimo grado de exactitud resulta fundamental para el éxito de la empresa.

Ciertamente, las universidades andaluzas ponen su énfasis en la transmisión de conocimientos formalizables, prestando escasa o nula atención a que esos otros conocimientos que estimulan o desarrollan capacidades y actitudes que se identifican con la empresarialidad sean transmitidos a los alumnos. Se explica así parte del limitado efecto que sobre la emergencia de empresarios ha tenido la educación en Andalucía, a la vez que se pone de manifiesto que el Sistema Educativo Andaluz debería introducir algunas reformas encaminadas a estimular, mediante la introducción de nuevas metodologías, la orientación de los alumuos hacia una actividad empresarial de calidad, sobre todo cuando se trate de estudios que se relacionen directamente con la actividad empresarial.

La conclusión anterior, que se ha construido a partir del análisis sobre la formación universitaria, toma mayor fuerza cuando además se observa el reducido porcentaje que representan los empresarios andaluces que iniciaron su actividad empresarial con titulación de Formación Profesional (tabla 2), lo que, como en el

8. García Echevarría, S. (1988): "El Empresario en la Sociedad Actual. Exigencias Desde la Perspectiva del managemet". El Empresario en la Sociedad Actual, II Congreso Mundial Vasco, pág. 36. 
caso anterior, sugiere que se están desaprovechando unas buenas posibilidades para estimular y fortalecer el tejido empresarial andaluz a través de la Formación Profesional.

\section{EPÍLOGO}

Dada la importancia que en los últimos años a se ha atribuido al empresario individual como factor capaz de generar riqueza y empleo, lo que puede enmarcarse en el auge que han tenido las políticas de desarrollo local, conocer los factores que favorecen o animan la aparición de empresarios y su calidad se ha convertido en una preocupación para muchos investigadores y políticos.

Entre un conjunto de factores que diversos autores han señalado como explicativos de la emergencia empresarial, el nivel de educación ha sido considerado en diferentes trabajos teóricos y empíricos como una variable que influye positivamente en la orientación de los individuos hacia el empresariado. No obstante, el efecto de esas influencias varia con el tipo de tipo de formación. Hemos señalado cómo, frente a lo que podríamos llamar formación "standard", existe otro tipo de formación específica que, con independencia de que transmita ciertos conocimientos formalizables, persigue desarrollar, mediante metodologías diferentes a las que se aplican en aquélla, capacidades y actitudes que se identifican con la empresarialidad.

Asimismo, hemos comprobado que en caso de Andalucía, pese a lo que establecen las aportaciones teóricas a las que anteriormente nos hemos referido, la educación no ha ejercido toda la influencia que cabría esperar en la emergencia empresarial. Los bajos niveles formativos de los empresarios emergidos en aproximadamente los últimos cuarenta años, así como el bajo nivel de titulaciones superiores y de Formación Profesional entre los que han creado su primera empresa en los últimos cinco años así lo evidencian. No obstante, esa carencia ha sido cubierta por la experiencia profesional previa de los empresarios emergidos, ya que casi en un $100 \%$ de los casos habían trabajado en algún tipo de actividad antes de poner en marcha su propio negocio. De esta forma, la empresa se ha convertido en una auténtica "academia para empresarios", hecho que tiene como ventaja que el aprendizaje se realiza por medio de la experiencia, pero que a su vez presenta entre otros inconvenientes el no ser el medio apropiado para la adquisición de ciertos conocimientos teóricos más sofisticados, lo que unido a la reducida dimen sión que las empresas tienen en Andalucía, limita considerablemente el aprendizaje que de esa forma se puede realizar.

Las universidades andaluzas ponen su énfasis fundamentalmente en la transmisión de conocimientos formalizables. De esa forma no se implican en la transmisión de otros conocimientos no formalizables que a diferencia de los anteriores 
estimulan y desarrollan capacidades y actitudes que se identifican con 1 i empresarialidad, lo que permite explicar, en parte, la limitada influencia de est: institución en la aparición de los empresarios andaluces. Esa conclusión toma fuer za si se consideran, junto a la Universidad, los Centros de Formación Profesiona pues, dado los elevados niveles de desempleo en Andalucía y las diversas rama! que en aquellos se imparten, llama la atención el reducido porcentaje que repre. sentan los empresarios andaluces con ese tipo de formación.

Por tanto, tomando como base las aportaciones teóricas y empíricas que poner de manifiesto que la oferta y calidad empresarial se incrementa a medida que cre. ce el nivel de formación, hemos de señalar que en Andalucía no se están aprove. chando todas las potencialidades que el Sistema Educativo ofrece para estimula la aparición de empresarios y contribuir así a solucionar uno de los problemas que en numerosas ocasiones ha sido manifestado por diversas instancias políticas y económicas: la debilidad del tejido empresarial.

\section{BIBLIOGRAFÍA}

DURING, W. (1990): "Education for Entrepreneurship", en Dockel, R. y Miettinen A. (eds.): New Findings and Perspective in Entrepreneurship. Avebury, England.

GARCÍA ECHEVARRÍA, S. (1988): "El Empresario en la Sociedad Actual. Exigencias Desde la Perspectiva del Managemet”. El Empresario en la Sociedad Actual, Il Congreso Mundial Vasco.

GONZÁLEZ SIMÓN, M. (1992): “Educación y Empresarios”. V1 Reunión ASEPELT-ESPAÑA, Granada.

GIBB, A. (1993): "The Enterprise Culture and Education. UnderstandingEnterprise Education ands its Links with Small Business. Entrepreneurship and Wider Educational Goals". International Small Business Journall núm. 11, vol. 3.

GUZMÁN, J. (1993): "La Calidad de la Empresa y del Empresario en Nuestro Entorno Económico", en la Empresa y el Empresario en el Desarrollo Económico. Fundación El Monte, Sevilla.

GUZMÁN, J. (1995): El Empresariado en la Provincia de Sevilla. Diputación Provincial de Sevilla, Sevilla.

JOHANNISSSON, B.(1991): "University Training for Entrepreneurship: Swedis Approaches". Entrepreneurship and Regional Development, núm. 3.

SARABIA, A. (1988): "La Universidad y su Influencia en el Desarrollo Empresarial". El empresario en la Sociedad Actual, Il Congreso Mundial Vasco.

SCHULTZ, T. (1985): Invirtiendo en la gente. Editorial Ariel, Barcelona. 